\title{
Wideband Gap-Waveguide Phase Shifter Based on a Glide- Symmetric Ridge
}

This paper was downloaded from TechRxiv (https://www.techrxiv.org).

\section{LICENSE}

CC BY-NC-SA 4.0

SUBMISSION DATE / POSTED DATE

$24-11-2021 / 08-12-2021$

\section{CITATION}

Palomares-Caballero, Ángel; Megías, Carlos; Molero, Carlos; Alex-Amor, Antonio; Padilla, Pablo (2021): Wideband Gap-Waveguide Phase Shifter Based on a Glide-Symmetric Ridge. TechRxiv. Preprint. https://doi.org/10.36227/techrxiv.17076164.v1

$\mathrm{DOI}$ 


\title{
Wideband Gap-Waveguide Phase Shifter Based on a Glide-Symmetric Ridge
}

\author{
Ángel Palomares-Caballero, Carlos Megías, Carlos Molero, Antonio Alex-Amor, and Pablo Padilla
}

\begin{abstract}
This paper presents a gap-waveguide phase shifter based on ridged unit cell with glide-symmetric configuration. The proposed unit cell design provides higher phase shift compared with a conventional ridged unit cell whose ridge height and waveguide width are tuned to achieve a stable phase shift. Through the insertion of glide-symmetric holes with semi-circle base in the ridged waveguide, a stable phase shift in a wide frequency range is achieved. Depending on the radii of the holes, the stable phase shift can be covered the desired frequency range. A $90^{\circ}$ phase shifter in millimeter-wave range is designed in order to validate the analysis. The impedance bandwidth of the phase shifter is from $32 \mathrm{GHz}$ to $42.5 \mathrm{GHz}(28.18 \%)$ providing a phase shift of $90^{\circ} \pm 2^{\circ}$ in the entire frequency range.
\end{abstract}

Index Terms-Phase shifter, ridge gap-waveguide, glide symmetry, millimeter-wave frequencies, wideband.

\section{INTRODUCTION}

$\mathbf{P}$ HASE shift in multi-beam antennas is a critical mechanism to achieve the adequate performance of this kind of radiating systems. One of the more attractive network to produce multi-beam radiation is the Butler matrix. For millimeter-wave applications, this beamforming network has been implemented in different waveguide technologies such as substrate integrated waveguide [1], rectangular waveguide [2], groove gap waveguide (GGW) [3] and printed ridge gap waveguide (PRGW) [4]. In all the previous works, a phase shifter in the corresponding technology has had to be designed. Ridge waveguide (RW) technology and its evolution in gap waveguide [5] have gained interest to develop millimeter-wave antennas [6] and multi-port components [7], [8]. Nevertheless, there are few reported ridge gap-waveguide (RGW) phase shifters in the literature. In [9] and [10] a RW phase shifter is designed based on a ridge with multiple steps and enlarging the length of the ridge, respectively. However, the phase shift is calculated considering a rectangular waveguide as a reference and gap-waveguide technology is not employed. The insertion

Manuscript submitted XX, YY. This work was supported in part by the Spanish Government under Project PID2020-112545RB-C54 and, Project RTI2018-102002-A-I00, in part by "Junta de Andalucía" under Project B-TIC402-UGR18, A-TIC-608-UGR20 and Project P18.RT.4830, and in part by the Predoctoral Grant FPU18/01965. (Corresponding author: Ángel PalomaresCaballero.)

Ángel Palomares-Caballero, Carlos Molero and Pablo Padilla are with the Departamento de Teoría de la Señal, Telemática y Comunicaciones, Universidad de Granada, 18071 Granada, Spain (e-mail: angelpc@ugr.es; cmoleroj@ugr.es; pablopadilla@ugr.es).

Carlos Megías is with the Department of Computer Architecture and Technology, CITIC, ETSIIT, University of Granada, 18071 Granada, Spain (email: narg@ugr.es)

Antonio Alex-Amor is with the Information Technologies Department, Universidad CEU San Pablo, 28003 Madrid, Spain (email: antonio.alexamor@ceu.es)

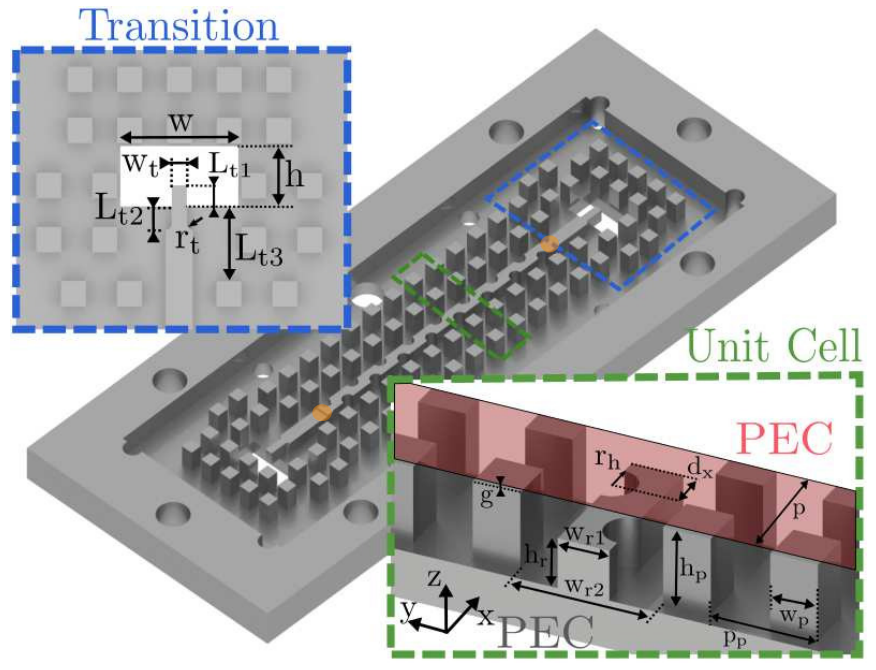

Fig. 1: Proposed gap-waveguide phase shifter with glide-symmetric ridged unit cells (framed in green). Planar view of the rectangular waveguide to ridge gap waveguide is framed in blue. Dimensions (units in $\mathrm{mm}$ ): $\mathrm{w}=5.69$, $\mathrm{h}=2.845, \mathrm{w}_{\mathrm{t}}=0.725, \mathrm{~L}_{\mathrm{t} 1}=1, \mathrm{~L}_{\mathrm{t} 2}=1.1, \mathrm{~L}_{\mathrm{t} 3}=3.5, \mathrm{r}_{\mathrm{t}}=0.3, \mathrm{p}=3.6$, $\mathrm{d}_{\mathrm{x}}=0.9, \mathrm{r}_{\mathrm{h}}=0.6, \mathrm{w}_{\mathrm{r} 1}=1.3, \mathrm{w}_{\mathrm{r} 2}=3.6, \mathrm{~h}_{\mathrm{r}}=1.55, \mathrm{~h}_{\mathrm{p}}=2.37, \mathrm{p}_{\mathrm{p}}=2.7$, $\mathrm{w}_{\mathrm{p}}=1.2, \mathrm{~g}=0.03$.

of materials with different permittivity and permeability in the RW is another option to introduce phase shift. In [11], the propagation constant is modified in the RW through the rotation of a dielectric slab placed atop the ridge. Liquid crystal (LC) is used in [12] in combination with gap-waveguide to properly bias the material but the phase shift response is not stable with the frequency. Materials with different relative permeability are also employed in the design of phase shifter in ridge gap waveguide [13]. Additionally, mechanical approach by taking advantage of the gap-waveguide technology is applied in [14] to vary the waveguide length. However, this implies bulk in the device.

Waveguide phase shifters loaded with pins [15] and holes [16] that preserve glide symmetry have demonstrated the benefits for producing phase shift in waveguides. This kind of higher symmetry greatly enhances the performance of radio-frequency devices [17]. Up to now, fully-metallic phase shifters have not been reported in RGW. In this document, we present a gap-waveguide phase shifter that exploits the use of ridged unit cells with glide symmetry to produce stable phase shift response over a wide bandwidth.

\section{GLide-SyMmetric RGW UNIT CELL}

The proposed ridge gap-waveguide phase shifter is illustrated in Fig. 1. It is composed by glide-symmetric ridged 
(GSR) waveguide unit cells (framed in green) and two rectangular waveguide to ridge gap-waveguide transitions inspired by [18] in order to design a back-to-back device. The electromagnetic bandgap (EBG) pin lattice provides a stopband from $12 \mathrm{GHz}$ to $61 \mathrm{GHz}$ that allows the proper operation of the RGW in the WR22 frequency range.

The illustrated ridged unit cell is the main responsible to produce the phase shift. The main difference of the proposed unit cell regarding a conventional RGW line [5] is the insertion of holes with semi-circle base placed along the x-direction. These holes present a glide-symmetric configuration where the symmetry plane corresponds to the XZ plane placed in the middle of the ridge and, the separation between hole centers is half of the unit cell period $\mathrm{p}$.

In order to study the phase shift behavior produced by a unit cell, dispersion diagrams are very useful to assess this characteristic [16]. Figs. 2(a) and 2(b) show the dispersion diagram and the phase shift of a conventional RGW unit cell. Note that for the computation of the phase shift, the reference RGW unit cell is the one whose dimensions are: $h_{r}=1.4 \mathrm{~mm}$ and, $\mathrm{w}_{\mathrm{r}}=3.6 \mathrm{~mm}$ (black line with circled markers). As the ridge height $h_{r}$ increases, the fundamental mode of the RGW unit cell lowers regarding to the reference RGW unit cell. This lowering of the modes in the dispersion diagram translates into an increase of the phase shift since the RGW unit cells with higher ridge height are more dense in terms of the propagation constant. However, the phase shifts obtained are not stable along the frequency range as can be observed in Fig. 2(b). On the other hand, another approach to introduce phase shift in a conventional RGW unit cell is by narrowing the width of the waveguide $\mathrm{w}_{\mathrm{r} 2}$ (green line with squared markers). In this case, the mode in the dispersion diagram goes up regarding the mode of the reference RGW unit cell. Contrary to the previous cases, the phase shift is negative because the RGW unit cell with narrow width is less dense than the reference RGW unit cell. A combination of both effects can be applied to achieve a RGW unit cell with stable phase shift along a wide frequency range (yellow line with downward-pointing triangles).

The use of GSR unit cells instead of conventional RGW unit cells with modified parameters provides a substantial advantage in the design of phase shifters. The proposed glidesymmetric configuration is the one that produces the largest bandwidth as it can been seen in the dispersion diagram of Fig. 2(c). If $d_{x}$ is not equal to a quarter of the period of the unit cell, the distance between hole centers is not equal to half of the unit cell period and a stopband around $42 \mathrm{GHz}$ appears. This implies a reduction of the operating frequency range and also, a loss of linearity of the propagating mode near the the stopband [19]. Note that the glide-symmetric configuration of the holes makes the stopband to vanish while the mirror-symmetric configuration $\left(\mathrm{d}_{\mathrm{x}}=\mathrm{p} / 2\right)$ provides the widest stopband according to the results discussed in [16].

Figs. 2(d) and 2(e) present the dispersion diagram and the produced phase shift when a GSR unit cell is analyzed. The ridge height of the GSR unit cell must be slightly higher than the reference RGW unit cell to achieve the desired effect of the glide-symmetric holes. The hole radii of the GSR unit cell are varied in order to observe the effects in the dispersion

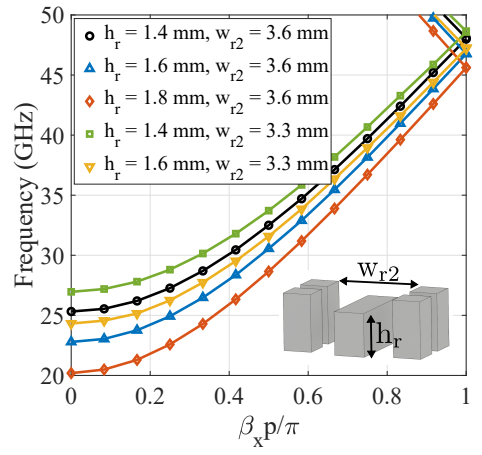

(a)

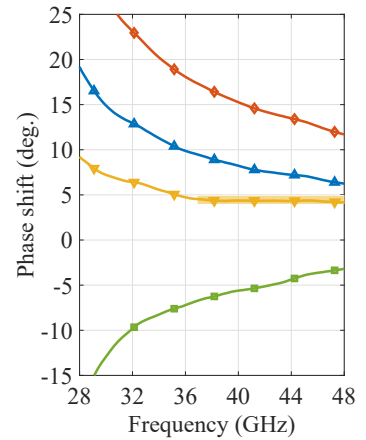

(b)

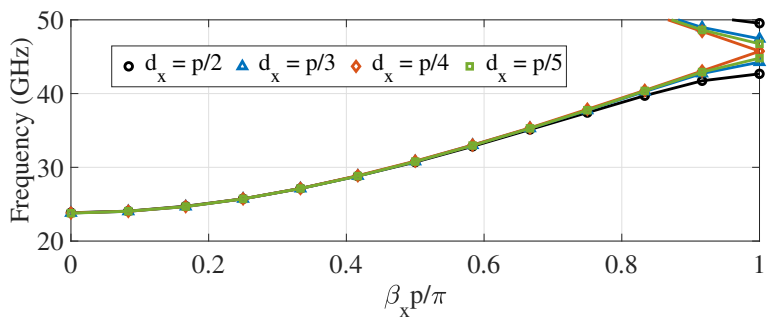

(c)

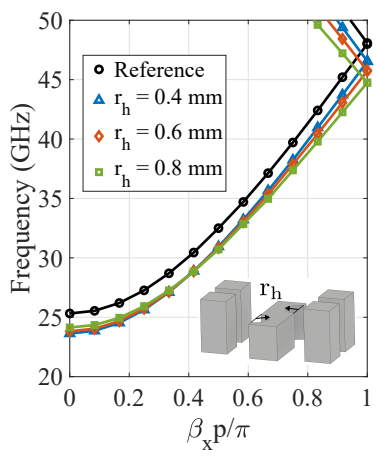

(d)

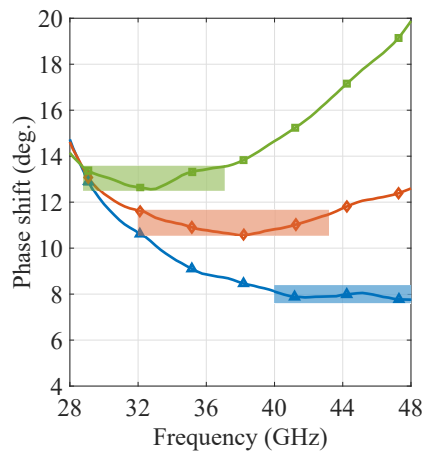

(e)
Fig. 2: (a) Dispersion diagram of a conventional RGW unit cell when the height and width of the ridge are varied. (b) Phase shift obtained from Fig. 2(a). (c) Dispersion diagram of the GSR unit cell when hole displacement $d_{x}$ is varied. (d) Dispersion diagram of the GSR unit cell when hole radius is varied. (e) Phase shift obtained from Fig. 2(d). To compute the phase shift, the reference RGW unit cell corresponds to $\mathrm{h}_{\mathrm{r}}=1.4 \mathrm{~mm}$ and $\mathrm{w}_{\mathrm{r} 2}=3.6 \mathrm{~mm}$. The other dimensions can be found in Fig. 1 .

diagram. When the glide-symmetric holes are introduced in the RGW unit cell, the unit cell becomes denser (higher equivalent refractive index) and the propagating propagation constant is therefore larger compared to the fundamental mode of the reference unit cell. Another important effect when the hole radii vary is the modification in the slope of the mode. For instance, as the hole radius increases, a lower slope is obtained in the dispersion diagram regarding of GSR unit cells. This interesting effect enables a stable and higher phase shift depending on the selected hole radius as illustrated in Fig. 2(e). In this figure, three different ranges of frequencies with phase-stable behavior are achieved. The smaller the radius, the smaller the phase shift produced by the unit cell but the produced phase shift is greater than the best case obtained in Fig. 2(b) (yellow line). Thus, the proposed GSR unit cell enables an easy way to produce a stable phase shift in the 


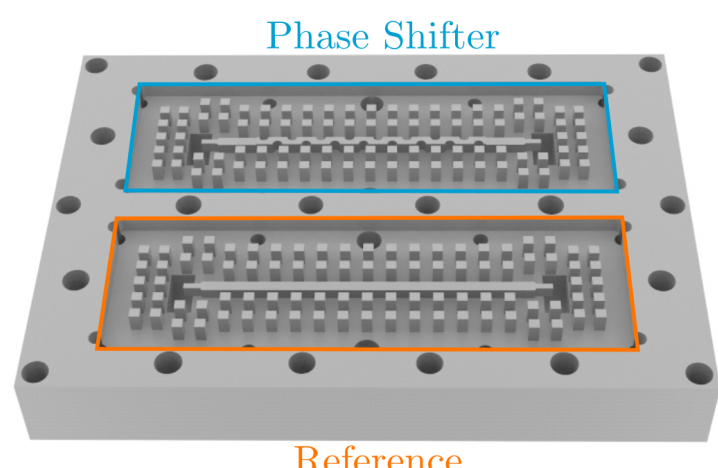

(a)

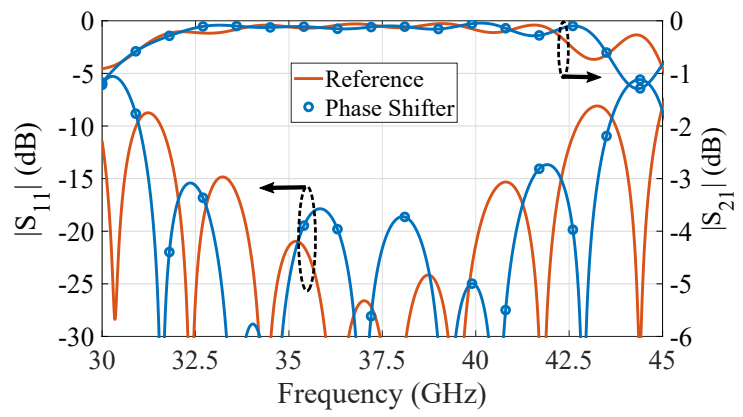

(b)

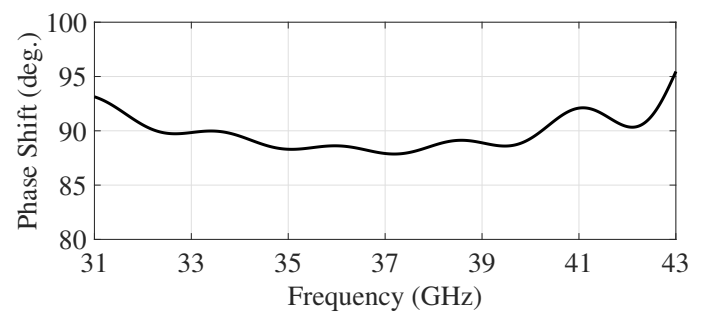

(c)

Fig. 3: RGW reference line and phase shifter: (a) 3-D view. (b) Magnitude of transmission and reflection parameters, and (c) phase shift.

desired frequency range.

\section{III. $90^{\circ}$ PHASE SHIFTER DESIGN}

Based on the proposed GSR unit cell, a $90^{\circ}$ phase shifter has been designed by the concatenation of eight unit cells. A RGW reference line with the same length of the phase shifter has also been designed in order to measure the produced phase shift. The dimensions of the unit cell employed in the RGW reference line correspond to the reference unit cell of Fig. 2(a). The back-to-back designs of the RGW reference line and phase shifter are illustrated in Fig. 3(a). The simulation of this design has been performed with CST Studio Suite where the metal considered in the structure is aluminum (losses are considered). In Fig. 3(b), the simulated transmission $\left|S_{21}\right|$ and reflection $\left|S_{11}\right|$ coefficients of the RGW reference line and phase shifter are shown. The common impedance bandwidth where $\left|\mathrm{S}_{11}\right|$ is below $-10 \mathrm{~dB}$ ranges from $32 \mathrm{GHz}$ to $42.5 \mathrm{GHz}(28.18 \%)$. In this frequency band, the difference output phase between the reference line and phase shifter is calculated and presented in Fig. 3(c). The achieved phase shift is approximately $90^{\circ} \pm 2^{\circ}$ in the bandwidth under consideration. It is important to note that this phase shift also includes the phase shift produced by the difference in ridge heights between the transitions and the concatenation of unit cells that form the phase shifter (orange areas highlighted in Fig. 1). This minimal discontinuity does not exist in the reference line design because there is no height difference in the ridge between the reference line and the transitions. Despite this negligible fact, phase shift achieved by the phase shifter is the one expected given the phase shift provided by a single RGW unit cell in the Fig. 2(e) (when $r_{h}=0.6 \mathrm{~mm}$ ).

Finally, Table I presents a performance comparison between the presented RGW phase shifter and other related waveguide phase shifters found in the literature. The proposed RGW phase shifter provides a wideband performance comparable to other state-of-the-art waveguide phase shifters. However, it is important to remark that this work presents the first (glidesymmetric) RGW phase shifter implementation that provides a stable phase shift response in wide operating frequency band.

TABLE I:

Comparison of waveguide phase shifters in mm-wave band with this work

\begin{tabular}{ccccc}
\hline Ref. & $\begin{array}{c}\text { Frequency } \\
(\mathbf{G H z})\end{array}$ & $\begin{array}{c}\text { Waveguide } \\
\text { Technology }\end{array}$ & $\begin{array}{c}\text { Max. IL } \\
(\mathbf{d B})\end{array}$ & $\begin{array}{c}\text { Stable } \\
\text { Phase Shift }\end{array}$ \\
\hline$[4]$ & $27-33.6(21.78 \%)$ & PRGW & 0.4 & Yes $\left(45^{\circ} \pm 2^{\circ}\right)$ \\
\hline$[10]$ & $33-50(40.1 \%)$ & RW & 9 & Yes $\left(90^{\circ} \pm 2^{\circ}\right)$ \\
\hline$[12]$ & $20-27(29.78 \%)$ & RGW and LC & 4.5 & No \\
\hline$[14]$ & $75-76.5(1.98 \%)$ & RGW & n.a. & No \\
\hline$[15]$ & $46-60(26.41 \%)$ & GGW & 1 & No \\
\hline$[16]$ & $56.5-74.5(27.5 \%)$ & GGW & 2 & Yes $\left(180^{\circ} \pm 5^{\circ}\right)$ \\
\hline This work & $32-42.5(28.18 \%)$ & RGW & 0.2 & Yes $\left(90^{\circ} \pm 2^{\circ}\right)$ \\
\hline
\end{tabular}

\section{Conclusion}

This paper presents a RGW phase shifter with a stable phase shift response in a wide frequency range. The phase shifter design is based on the use of ridged unit cells modified by the inclusion in their structure of glide-symmetric holes with semi-circle base. By tuning the radii of the glide-symmetric holes and the height of the ridge of the unit cell, a stable phase shift can be achieved in a desired frequency range. As a proof of concept, a $90^{\circ}$ phase shifter is designed by the concatenation of glide-symmetric ridged unit cells. The simulated results reveal a low loss and wideband phase shifter whose produced phase shift is $90^{\circ} \pm 2^{\circ}$ from $32 \mathrm{GHz}$ to 42.5 $\mathrm{GHz}$ (impedance bandwidth of $28.18 \%$ ). The presented design has been prototyped and experimental results are expected to be included soon. The proposed RGW phase shifter enables the potential development of cost-effective Butler matrix in ridge waveguide technology that are lacking in the literature.

\section{REFERENCES}

[1] Y. Cao, K. Chin, W. Che, W. Yang and E. S. Li, "A Compact 38 $\mathrm{GHz}$ Multibeam Antenna Array With Multifolded Butler Matrix for 5G Applications," IEEE Antennas Wirel.Propag. Lett., vol. 16, pp. 29962999, 2017.

[2] K. Tekkouk, J. Hirokawa, R. Sauleau, M. Ettorre, M. Sano and M. Ando, "Dual-Layer Ridged Waveguide Slot Array Fed by a Butler Matrix With Sidelobe Control in the 60-GHz Band," IEEE Trans. Antennas Propag., vol. 63, no. 9, pp. 3857-3867, Sept. 2015. 
[3] A. Tamayo-Domínguez, J. Fernández-González and M. Sierra-Castañer, "3-D-Printed Modified Butler Matrix Based on Gap Waveguide at WBand for Monopulse Radar," IEEE Trans. Microw. Theory Techn., vol. 68, no. 3, pp. 926-938, March 2020.

[4] I. Afifi and A. -R. Sebak, "Wideband $4 \times 4$ Butler Matrix in the Printed Ridge Gap Waveguide Technology for Millimeter-Wave Applications," IEEE Trans. Antennas Propag., vol. 68, no. 11, pp. 7670-7675, Nov. 2020 ,

[5] E. Rajo-Iglesias, M. Ferrando-Rocher and A. U. Zaman, "Gap Waveguide Technology for Millimeter-Wave Antenna Systems," IEEE Commun. Mag., vol. 56, no. 7, pp. 14-20, July 2018.

[6] J. Liu, A. Vosoogh, A. U. Zaman and J. Yang, "A Slot Array Antenna With Single-Layered Corporate-Feed Based on Ridge Gap Waveguide in the $60 \mathrm{GHz}$ Band," IEEE Trans. Antennas Propag., vol. 67, no. 3 , pp. 1650-1658, March 2019.

[7] A. Beltayib and A. Sebak, "Analytical Design Procedure for Forward Wave Couplers in RGW Technology Based on Hybrid PEC/PMC Waveguide Model," IEEE Access, vol. 7, pp. 119319-119331, 2019.

[8] A. Farahbakhsh, "Ka-Band Coplanar Magic-T Based on Gap Waveguide Technology," IEEE Microw. Wireless Compon. Lett., vol. 30, no. 9, pp. 853-856, Sept. 2020.

[9] J. L. Cano, A. Mediavilla, and A. Tribak, "Parametric design of a class of full-band waveguide differential phase shifters," Electronics ,vol.8,no. 3, p. 346, Mar. 2019

[10] E. Villa, B. Aja, J. Cagigas, E. Artal and L. de la Fuente, "Four-State Full Q-Band Phase Shifter Using Smooth-Ridged Waveguides," IEEE Microw. Wireless Compon. Lett., vol. 27, no. 11, pp. 995-997, Nov. 2017.

[11] A. Ghasemi and J. Laurin, "Beam Steering in Narrow-Wall Slotted Ridge Waveguide Antenna Using a Rotating Dielectric Slab," IEEE Antennas Wirel. Propag. Lett., vol. 17, no. 10, pp. 1773-1777, Oct. 2018.

[12] M. Nickel et al., "Ridge Gap Waveguide Based Liquid Crystal Phase Shifter," IEEE Access, vol. 8, pp. 77833-77842, 2020.

[13] M. A. Abdelaal, S. I. Shams and A. A. Kishk, "Compact RGW Differential Phase Shifter for Millimeter-Wave Applications," in 2018 18th International Symposium on Antenna Technology and Applied Electromagnetics (ANTEM), Waterloo, ON, 2018.

[14] H. Kirino and K. Ogawa, "A $76 \mathrm{GHz}$ phased array antenna using a waffle-iron ridge waveguide," in Proceedings of the Fourth European Conference on Antennas and Propagation, Barcelona, 2010.

[15] Á. Palomares-Caballero, A. Alex-Amor, P. Padilla, F. Luna and J. Valenzuela-Valdes, "Compact and Low-Loss V-Band Waveguide Phase Shifter Based on Glide-Symmetric Pin Configuration," IEEE Access, vol. 7, pp. 31297-31304, 2019.

[16] Á. Palomares-Caballero, A. Alex-Amor, P. Padilla and J. F. ValenzuelaValdés, "Dispersion and Filtering Properties of Rectangular Waveguides Loaded With Holey Structures," IEEE Trans. Microw. Theory Techn., vol. 68 , no. 12 , pp. 5132-5144, Dec. 2020

[17] O. Quevedo-Teruel, Q. Chen, F. Mesa, N. J. G. Fonseca, G. Valerio, "On the benefits of glide symmetries for microwave devices," IEEE Journal of Microwaves, vol. 1, no. 1, pp. 457-469, winter 2021.

[18] A. U. Zaman and P. Kildal, "Wide-Band Slot Antenna Arrays With Single-Layer Corporate-Feed Network in Ridge Gap Waveguide Technology," IEEE Trans. Antennas Propag., vol. 62, no. 6, pp. 2992-3001, June 2014.

[19] O. Quevedo-Teruel, M. Ebrahimpouri and M. Ng Mou Kehn, "Ultrawideband Metasurface Lenses Based on Off-Shifted Opposite Layers," IEEE Antennas Wirel. Propag. Lett., vol. 15, pp. 484-487, Dec. 2016. 